\title{
Visages. Les Peuples de l'Afrique orientale
}

Berlin, Europrint, 2014, 472 p., bibl., cartes, dessins et photographies de l'auteur

\section{Serge Tornay}

\section{Q OpenEdition}

1 Journals

Édition électronique

URL : http://journals.openedition.org//homme/28910

DOI : 10.4000/lhomme.28910

ISSN : 1953-8103

Éditeur

Éditions de l'EHESS

\section{Édition imprimée}

Date de publication : 25 février 2016

ISSN : 0439-4216

\section{Référence électronique}

Serge Tornay, «Visages. Les Peuples de l'Afrique orientale », L'Homme [En ligne], 217 | 2016, mis en ligne le 24 février 2018, consulté le 23 septembre 2020. URL : http://journals.openedition.org/lhomme/ 28910 ; DOI : https://doi.org/10.4000//homme.28910

Ce document a été généré automatiquement le 23 septembre 2020.

(c) École des hautes études en sciences sociales 


\section{Visages. Les Peuples de l'Afrique orientale}

Berlin, Europrint, 2014, 472 p., bibl., cartes, dessins et photographies de l'auteur

Serge Tornay

Christian Bader, Visages. Les Peuples de l'Afrique orientale, Berlin, Europrint, 2014, 472 p., bibl., cartes, dessins et photographies de l'auteur

1 EN GUISE d'introduction, on ne peut mieux faire que citer l'auteur dans la quatrième de couverture de son ouvrage: «Ces Visages sont ceux de l'Afrique que j'ai vue et aimée tout au long de vingt-cinq années d'expéditions, de bivouacs et de pistes parcourues à pied, à dos de mule ou en voiture, à travers les régions les plus reculées de l'Afrique orientale, à la recherche de ces peuples qui, comme surgis des premiers matins du monde, sont parvenus tant bien que mal à préserver jusqu'à aujourd'hui leurs antiques coutumes, leurs langues dont certaines restent à peu près inconnues, leurs rites parfois étranges, leurs fascinantes et singulières parures ».

On a connu, on connaît des diplomates écrivains, mais combien d'entre eux se sont-ils illustrés comme anthropologues? Victor Segalen n'était pas diplomate mais médecin militaire, et ses Immémoriaux, écrits à Tahiti entre 1902 et 1905, furent publiés en 1956 dans la collection "Terre humaine », avec la dédicace « aux Maori des temps oubliés » ${ }^{57}$. L'éditeur présentait l'ouvrage comme un roman ethnographique, à la différence de Visages. Les Peuples de l'Afrique orientale qui n'est pas un roman. C'est le témoignage d'un grand voyageur devenu ambassadeur, non seulement de France, mais d'un nombre impressionnant de peuples, petits ou grands, qui font la richesse culturelle des vastes confins de l'Éthiopie, du Soudan, du Kenya et de l'Ouganda, un champ d'exploration qu'aucun ethnologue n'a parcouru avec autant de constance et de passion, une aventure synthétisée dans une publication à tous égards exceptionnelle. 
3 Visages serait-il un "beau livre» de plus? C'est certes un beau livre, au format inhabituel et réalisé avec le plus grand soin : c'est un écrin digne de recevoir l'une des substances du livre, à savoir les riches photographies, plus de quatre cents, consacrées à la trentaine de peuples explorés. Femmes, hommes, jeunes et vieux, tous en majesté. Il y a des adolescents, mais peu d'enfants, sinon sur le dos ou la hanche de leur mère. Les portraits sont privilégiés, allant du visage à la personne en pied : chaque photo met en valeur un style, une empreinte culturelle que chaque peuple décline, avec une inventivité étonnante, en particulier pour la gent féminine. Les visages, comme les poitrines, les épaules, les oreilles et d'autres parties du corps, sont marqués par des scarifications qui s'offrent comme l'affirmation d'une identité, affirmation corroborée par les coiffures, les colliers et bracelets de verroteries, de noyaux comme ceux des jujubes, d'autres éléments de bois, de coquille d'œufs d'autruche, d'ivoire, d'os, de métal, les labrets, les jupes et cache-sexe de peaux, parures rehaussées des mêmes ornements. Christian Bader recrée pour nous cette Afrique orientale qui a fasciné et qui fascine encore, tout au moins là où l'on peut l'entrevoir non encore frelatée sous les avancées du tourisme international et, plus globalement, de ce qu'on nomme la mondialisation. L'auteur est un infatigable arpenteur des solitudes qui paie, souvent très cher, de sa personne pour aller dénicher des témoignages au cœur de cette Afrique sans masques, comme l'a nommée ailleurs l'auteur de ces lignes ${ }^{58}$. Oui, cette humanité a découvert que la beauté des corps était l'arme la plus sûre contre l'ennui et le dénuement. La vaste fresque de ces Visages est inégalée et elle demeurera unique car les temps qui viennent vont inexorablement l'oblitérer. Pour citer encore brièvement notre auteur dans la quatrième de couverture : «Ce livre est dédié à ces hommes et à ces femmes qui m'ont offert, le temps d'un voyage toujours trop court, invitation perpétuelle à d'autres voyages, de partager un peu de leur âpre existence et de devenir, certes moins que l'un des leurs, mais mieux qu'un étranger : un hôte, invité à assister à un culte auquel sacrifient ces portraits capturés par la caméra, et dont quelques-uns appartiennent déjà à des temps révolus : le culte opiniâtre qu'ils vouent, depuis le fond des âges et avec une fierté rarement entamée, à la Beauté ».

4 L'ouvrage est construit avec une méthode qui en fait un véritable outil de travail et de référence. Chaque chapitre, qui se donne comme clé le dessin de l'appuie-tête caractéristique du peuple considéré, accorde une place de choix aux cheminements qui ont permis, au terme d'aventures parfois rocambolesques, toujours risquées, la rencontre entre une communauté et notre voyageur, qui n'a de cesse de recueillir les données linguistiques et ethnographiques que les personnages, si présents dans les photographies, toutes réalisées dans un climat de confiance mutuelle, ont le sentiment de livrer comme une sorte de testament : voilà ce que nous avons été, voilà ce que nous sommes, mais pour combien de temps encore? Chaque peuple est replacé dans son contexte géographique par une carte originale, que l'auteur a tenu à réaliser de sa main. Enfin, chaque chapitre contient un encadré, un précieux aide-mémoire qui permet de saisir d'un coup d'œil des données essentielles: État où vit ce peuple, population ou démographie, localités nommées, classes d'âge ou autres groupes sociaux nommés, religion, mode de subsistance, famille linguistique, ethnies voisines et autres noms donnés au groupe étudié. De sorte que l'ouvrage est le plus somptueux des Who Is Who de cette partie de l'Afrique orientale, dont il nous donne à scruter l'identité. La bibliographie qui clôt le volume est la plus exhaustive, sur ces trente populations, qui ait jamais été réunie et publiée. 
Il est utile de nommer les groupes ethniques dont les vingt-trois chapitres du livre nous font le minutieux portrait. Certains de ces groupes sont connus des ethnologues et parfois du grand public, d'autres beaucoup moins, voire pas du tout. Pour l'Éthiopie : les Ongota, moins de cent personnes, dont 4 ou 5 locuteurs seulement de langue ongota ; les Kaara, 1250 personnes ; les Arbore, 4500 ; les Bodi, 7000 ; les Mursi, 7500 ; les Ts'amakko, 11000 ; les Suuri et Baale, 9000 (5000 en Éthiopie, 4000 au Soudan); les Nyangatom, 14200 ; les Hamar, 20000 ; les Chai et les Tirma, 22700 ; les Banna, 23000 ; les Daasanech, 39000 ; et les Tishana, 55000 personnes. Pour le Sud-Soudan: les Tennet, 4000 représentants ; les Jiye, 9 à 10000 et les Toposa, 110 à 150000 personnes ; les Murle (sans estimation de leur population); les Larim, 20000 ; les Didinga, 60000 personnes. Pour le Kenya : les Elmolo, 150 personnes ; les Rendile, 34700 ; les Pokot, 300 000 personnes (250 000 au Kenya, 50000 en Ouganda); les Turkana 455000 personnes. Pour l'Ouganda : les Ik, 4500 individus et les Soo, 3500 ; les Karimojong, 330000 ; les Jie, 50000 ; et les Dodos, 60000 personnes. Plusieurs groupes en voie de disparition sont sauvés de l'oubli grâce à cet ouvrage : par exemple, les Ongota d'Éthiopie, les Elmolo du Kenya, les Ik et les Soo d'Ouganda. L'histoire de ces groupes «miraculés » est émouvante, et elle doit demeurer le privilège des lecteurs.

6 La mère de l'ethnographie est la linguistique, plus précisément l'ethnolinguistique qui met en pratique la parenté des deux disciplines. Christian Bader s'y est exercé, d'abord en Alsace, sa région d'origine. La publication du Lexique des parlers sundgauviens ${ }^{59}$ est sans doute celle d'un manuscrit qui doit remonter à sa jeunesse. La Namibie, avec son désert et un univers végétal et animal des plus singuliers, n'a pas manqué d'éveiller la curiosité de notre explorateur pour les Himba, pasteurs nomades matrilinéaires, et leurs moutons damara dont les robes colorées sont uniques au monde ${ }^{60}$. Une série de publications vient ensuite confirmer l'ancrage en Afrique orientale: Le Sang et le lait. Brève histoire des clans somali ${ }^{61}$, Mythes et légendes de la Corne de l'Afrique $e^{62}$, Les Yibro, mages somali. Les juifs oubliés de la Corne d'Afrique, Les Noms de personnes chez les Somali63. Mentionnons encore : Yéniches. Les derniers nomades d'Europe et Parlons oromo. Une langue de la Corne de l'Afrique ${ }^{64}$. Les Guerriers nus ${ }^{65}$, une aventure à couper le souffle aux confins de l'Éthiopie et du Soudan, ainsi que Parlons karimojong. Une langue de l'Afrique orientale ${ }^{66}$ sont deux livres qui nous font entrer de plain-pied dans le domaine de Visages.

7 La grande fresque de Christian Bader explore un territoire considérable. Du pays éthiopien des Tishana au nord à celui des Pokot du Kenya au sud, la distance est d'environ $600 \mathrm{~km}$. D'est en ouest, du pays des Ongota en Éthiopie et de celui des Rendile au Kenya, jusqu'à celui des Murle, des Didinga, des Larim et des Tennet au Sud-Soudan, et en allant jusqu'aux Karimojong, aux Jie, aux Dodos, aux Ik et aux Soo d'Ouganda, la longitude se déploie sur au moins $500 \mathrm{~km}$. Au total, c'est un espace de $300000 \mathrm{~km}^{2}$ de savanes arides, souvent rocailleuses et désertiques, à cheval sur quatre États d'Afrique orientale que l'auteur a parcourus avec une constance qui n'a pas faibli au cours des trois dernières décennies.

8 En dépit de la diversité de leurs origines et de leurs histoires culturelles, les personnages du livre ont comme un air de parenté. Quelle serait l'énigme qui se cache derrière cette spécificité ? On doit la reconnaître en premier lieu au plan géographique. La première carte du livre met en évidence un espace, non défini en tant qu'aire culturelle dans la littérature, qui réunit les confins de l'Éthiopie méridionale avec les territoires adjacents du Sud-Soudan, lesquels ont leurs prolongements au Kenya du nord-ouest et dans l'Ouganda nord-oriental. Dans cette vaste zone qui porte bien son nom de confins, les 
peuples réunis dans Visages sont tous voisins, sans interstices pour des corps étrangers à leur monde. Il s'agit d'un ensemble d'agropasteurs de langues nilotiques, surmiques et kuliak pour le Soudan et le nord-ouest du Kenya, de langues nilotiques, omotiques et, pour une part, couchitiques pour l'Éthiopie du Sud-Ouest, sans oublier la langue "isolée ", bien énigmatique, des Ongota. Les peuples de langues bantu du Kenya, de langues amhariques, oromo et somali de la grande Éthiopie sont restés à l'écart du domaine délimité par le livre de Christian Bader. Les contiguïtés géographiques ne sauraient cependant, à elles seules, expliquer cet étrange air de parenté qui émane de ces Visages réunis pour la première fois par l'intuition d'un observateur érudit et passionné.

9 La sauvegarde, par les acteurs eux-mêmes, d'un patrimoine culturel d'une telle richesse, dans sa variété affirmée sur des fonds partagés, pourrait-elle être due à l'état d'isolement de ces régions que le colonialisme, pas plus que l'impérialisme ou d'autres formes de domination n'ont pu éroder? Cet isolement a joué un rôle, pas de façon négative par la simple érection de barrières, mais d'une manière constructive, en créant un espace d'autonomie, contraignante tout autant que choisie, un espace de liberté : ces peuples ont pratiqué et pratiquent encore l'art de ne pas être gouvernés. Cette expression est le titre d'un livre qui a attiré l'attention dès sa publication, The Art of Not Being Governed de James C. Scott ${ }^{67}$. L'ouvrage a été traduit en français en 2013 sous le titre Zomia ou l'Art de ne pas être gouverné6 ${ }^{6}$. On lit dans la préface de l'auteur : "Zomia est un terme récent employé pour désigner grosso modo tous les territoires situés à des altitudes supérieures à 300 mètres, des hautes vallées du Vietnam aux régions du nordest de l'Inde, et traversant cinq pays d'Asie du Sud-Est ainsi que quatre provinces chinoises [...]. La thèse que je défends ici est à la fois simple, osée, et polémique. La Zomia est la dernière région $d u$ monde dont les peuples n'ont pas encore été complètement intégrés à des États-nations. Ses jours sont comptés ». La thèse de James C. Scott se réclame ouvertement de La Société contre l'État de Pierre Clastres ${ }^{69}$. Dans les études africaines et plus spécifiquement éthiopiennes, ce sont des collègues de l'Institut Max Planck de Halle qui ont pris les devants d'une nouvelle réflexion comparative sur les sociétés acéphales vivant dans les marges de certains pouvoirs étatiques. Günther Schlee, Felix Girke et Immo Eulenberger ont organisé, en février 2013 à Halle, une table ronde intitulée "Inter-Related Conflicts in a Northeast African Border Region». Le sous-titre, "Azomia 1 ", laisse entendre que la thèse de Scott a de l'avenir non seulement pour l'Asie, mais bel et bien pour l'Afrique, en l'occurrence cette Afrique orientale que l'ouvrage de Christian Bader nous donne à penser. La carte dessinée pour la rencontre de Halle recouvre assez exactement celle de Bader dans Visages : ce sont bien les confins de l'Éthiopie méridionale et du Sud-Soudan adjacent, du Kenya nordoccidental et de l'Ouganda voisin. S'agit-il d'un simple hasard ? Nous ne le pensons pas.

Felix Girke a présenté, dans une Rencontre organisée à Leiden par Jon Abbink en novembre 2011 sur le thème "Rethinking the Anthropology of Ethiopia », une réflexion intitulée "Over the Hills and Far Away: The Art of Not Being Governed in Ethiopia ». Cette contribution reconsidère les conceptions classiques de l'Éthiopie par des auteurs comme Harold Fleming, Donald Levine, John Markakis ou Igor Kopytoff à la lumière de l'essai de James Scott sur l'Asie du Sud-Est. Des notions essentielles comme les frontières, zones refuge ou de fragmentation, y sont soigneusement examinées, en tenant compte du fait que la Zomia, en Asie du Sud-Est, est celle des hauteurs et que ce schéma est inversé en Éthiopie du fait que le pouvoir étatique s'est développé sur les Hauts-Plateaux. Mais le parallèle avec l'Asie du Sud-Est demeure pertinent. Voici la 
façon dont Scott, dans de courts extraits choisis par Girke et traduits par nous en français, décrit la Zomia asiatique. Des groupes ont cherché refuge dans une périphérie non gouvernée, « une périphérie composée tout autant de réfugiés que de groupes qui n'ont jamais été sujets d'un État. Ces régions marginales offraient des opportunités à ceux qui les choisissaient, mais aussi au pouvoir qui ne parvenait pas à les dominer : insoumis, peu dociles et relativement peu organisés, les gens qui se gouvernent euxmêmes pouvaient être victimes de razzias et de captures esclavagistes par les forces militaires supérieures d'un centre étatique, mais leur style de vie représentait une tentation permanente, celle d'une alternative à la vie sous un État. Ces peuples sans État n'étaient pas enchâssés dans un système de salariat agricole. La civilisation avait peu d'attrait pour eux dans la mesure où ils pouvaient prospérer sans connaître le statut d'hommes de peine, de tâcherons, la subordination et l'immobilité des sujets d'un État. La structure sociale comme la technique agricole ne sont pas des donnés immédiats, mais des choix opérés sur le long terme et de nature politique. Quand une société ou l'une de ses parties choisit d'échapper à l'incorporation ou à l'appropriation, elle s'oriente vers des unités sociales plus simples, plus petites et plus dispersées. Si l'agriculture itinérante sur brûlis et la dispersion des communautés sont des stratégies qui empêchent la mainmise d'un pouvoir, si la fragmentation sociale et l'organisation acéphale font obstacle à l'incorporation étatique, alors, du même coup, l'absence d'écriture et donc de textes facilite, par manque de preuves opposables parce que lisibles, des libertés qui entravent les routines étatiques ${ }^{70}$. Pour soutenir que les vues de Scott sont applicables à une Zomia éthiopienne, Felix Girke fait trois brèves allusions à la basse vallée de l'Omo ${ }^{71}$. Dès les années 1970, Ivo Strecker avait noté que les Hamar avaient changé leur mode de subsistance après la conquête éthiopienne pour s'appuyer plutôt sur le pastoralisme nomade que sur l'agriculture sédentaire, afin de se soustraire à une exploitation systématique. Et cela en dépit du fait qu'ils avaient un profond attachement à l'agriculture. Il est surprenant que les groupes de descendance des Kara, petite communauté de la rive orientale de l'Omo, n'aient pas de profondeur : les aïeux de la troisième génération sont déjà considérés comme non pertinents ou simplement inconnus. Ce fait est en accord avec la brève ethnohistoire des Kara selon laquelle leurs prédécesseurs sont arrivés dans la basse vallée de l'Omo en tant que dissidents de groupes omotiques plus importants qui habitaient les piémonts éthiopiens. Les Arbore ont besoin de miel pour des rites essentiels et, jusqu'à récemment, ils ont refusé d'en acquérir par échange avec les Hamar ou les Borana, des alliés trop incertains. Ils font le choix de se tenir à distance de leurs voisins pour affirmer leur identité et assurer leur autonomie. Ces simples exemples suggèrent que le modèle des zones de fragmentation postulé par James C. Scott pour les hauteurs de l'Asie du Sud-Est possède un fort potentiel d'application à l'Éthiopie du Sud.

11 Ces réflexions nous ramènent aux Visages de Christian Bader. Qu'est-ce qui a guidé l'auteur dans le choix des personnes et des communautés à qui il rend hommage par son livre? Même si ce n'est pas un aveu explicite, on devine sans peine que ce qui a fasciné notre auteur-diplomate, rompu aux règles et contraintes des États wébériens ceux qui existent par l'affirmation, et au besoin par le recours à la guerre, d'une souveraineté sans partage sur un territoire délimité par des frontières -, est d'avoir rencontré des peuples dont l'idéal est d'ignorer les frontières, de se mouvoir pour s'affranchir de toute servitude, en particulier de celle des États, qui demeurent pour eux des corps étrangers. Christian Bader s'est fait l'ambassadeur des peuples d'avant, dont la survie et peut-être même la mémoire, nous le savons, sont désormais menacées. 


\section{NOTES}

57. Paris, Plon, 1956.

58. Notamment, dans Serge Tornay, «Une Afrique démasquée : initiation et sacrifice chez les Pasteurs d'Afrique orientale ", in Pierre Centlivres \& Jacques Hainard, eds, Les Rites de passage aujourd'hui, Lausanne, L'Âge d'homme, 1986 : 69-92.

59. Mulhouse, Éd. du Rhin, 1997.

60. La Namibie, Paris, Karthala, 1997 (« Méridiens »).

61. Paris, Maisonneuve \& Larose, 1999.

62. Paris, Karthala, 2000 («Contes et légendes »).

63. Ces deux derniers ouvrages ont été publiés chez L'Harmattan, respectivement en 2000 et 2004 .

64. Tous deux publiés chez L'Harmattan en 2007, le second dans la collection « Parlons ».

65. Paris, Payot, 2002.

66. Paris, L’Harmattan, 2008 (« Parlons »).

67. James C. Scott, The Art of Not Being Governed. An Anarchist History of Upland Southeast Asia, New Haven, Yale University Press, 2009.

68. Trad. de l'anglais par Nicolas Guilhot, Frédéric Joly et Olivier Ruchet, Paris, Le Seuil, 2013.

69. Pierre Clastres, La Société contre l'État. Recherches d'anthropologie politique, Paris, Minuit, 1974 ( «Critique »).

70. James C. Scott, The Art of Not Being Governed..., op. cit. : x (trad. Serge Tornay).

71. Des analyses développées dans Homeland, Boundary, Resource. The Collision of PlaceMaking Projects on the Lower Omo River, Ethiopia, Halle, Max Planck Institute for Social Anthropology, 2013 : 3 (Working Paper n¹48). 